\title{
Aprender a mirar con sentido: facilitar la interpretación de las interacciones en el aula
}

\author{
J. M. Fortuny y R. Rodríguez \\ Universitat Autònoma de Barcelona (España)
}

Recibido el 20 de Julio de 2012; aceptado el 8 de Septiembre de 2011

Aprender a mirar con sentido: facilitar la interpretación de las interacciones en el aula

\section{Resumen}

En este artículo se presenta una investigación sobre cómo los profesores pueden aprenden a mirar con sentido e interpretar las interacciones de clase. Definimos lo que significa "mirar con sentido" las interacciones en el aula y describimos un instrumento de video relatos para aprender a hacerlo.

Descriptores. Aprendizaje, didáctica de la matemática, formación de profesorado.

Aprender a notificar e a interpretar as interacções da aula

Resumo

Neste artigo apresenta-se uma investigação sobre como os professores podem aprender a notificar $e$ a interpretar as interacções da aula. Definimos o que significa "notificar" interacções da aula e descrevemos um instrumento de vídeo-relatos para aprender a fazê-lo.

Palavras Chave. Aprendizagem, didáctica da matemática, formação de professores

\section{Learn to notice and interpret classroom interactions}

Summary

This article presents research on how teachers can learn to notice and interpret classroom interactions. Define what "report" interactions in the classroom and describe an instrument of video stories to learn how

Key words. Mathematics education, teacher training.

Apprendre à notifier et interpréter les interactions de classe

\section{Résumé}

Dans cet article nous présentons une recherche qui étudie comment les professeurs peuvent apprendre à notifier et interpréter les interactions de classe. Nous définissons ce que signifie "notifier" et nous décrivons un instrument de vidéo qui permettra d'apprendre à le faire.

Paroles clés. Apprentissage, didactique de la mathématique, formation de professeurs

Para citar: Fortuny, J.M., \& Rodríguez, R. (2012). Aprender a mirar con sentido: facilitar la interpretación de las interacciones en el aula. Avances de Investigación en Educación Matemática, 1, 23 - 37

(C) Sociedad Española de Investigación en Educación Matemática (SEIEM). www.seiem.es 
Los planes de capacitación docente contemplan en la formación inicial la realización de diferentes períodos de prácticas, resaltando que formar profesores es un asunto eminentemente práctico. Por este motivo es relevante considerar el proceso de formación práctica del estudiante en su aprendizaje para enseñar matemáticas. Consideramos a los estudiantes para profesor como individuos, conjuntamente con los procesos de cambio y desarrollo, que son constitutivos del proceso de llegar a ser un profesor.

El llegar a ser un profesor implica saber observar de manera profesional los fenómenos que ocurren en una situación docente, en especial identificar, interpretar y valorar aprendizajes específicos del alumnado. Así mismo, usar los conocimientos de didáctica de las matemáticas para presentar alternativas para mejorar la calidad de la enseñanza. La diferencia con un profesional docente, reside en que la observación de un fenómeno docente se queda en una mera descripción anecdótica.

Esta visión se plantea desde la complejidad del proceso de generación de las competencias necesarias para enseñar, contemplando el carácter integrador de los diferentes dominios de conocimientos del profesor y la necesaria articulación del conocimiento aprendido en la universidad con el conocimiento generado en la práctica (Llinares \&Valls, 2009).

Sostenemos que los estudiantes para profesor de matemáticas pueden desarrollar respuestas a nuevas formas de percibir e interpretar las interacciones en el aula. Sin embargo, los actuales programas de formación del profesorado, a menudo, no se centran en ayudar a los profesores a interpretar las interacciones en el aula. En su lugar, se concentran en ayudar al profesorado en formación a actuar, y solo se les proporcionan nuevas técnicas pedagógicas, tecnología y nuevas actividades que pueden utilizar. Aunque estas técnicas y actividades son sin duda importantes recursos para el profesorado en formación, no garantizan necesariamente que los estudiantes para profesor de matemáticas interpreten las interacciones en el aula, de manera que permitan una flexibilidad en su enfoque de la enseñanza. Es decir, pueden apoyar un cambio didáctico en su actuación, pero no llegar a interiorizar en su pensamiento docente un cambio profesional.

En este artículo se presenta una propuesta sobre cómo los profesores pueden aprenden a mirar con sentido e interpretar las interacciones de clase. Definimos lo que significa mirar con sentido las interacciones en el aula y describimos un instrumento de vídeo relatos para aprender a hacerlo.

\section{Aprender a mirar con sentido}

Nuestro estudio se enmarca en dos aspectos principales. En primer lugar, definimos lo que entendemos por la capacidad de los docentes de mirar con sentido las interacciones del aula. En segundo lugar, dirigimos la investigación sobre el uso del vídeo-relato en la formación del docente, considerando las razones por las que puede ser un instrumento útil para apoyar y contemplar los esfuerzos del profesorado en formación a mirar con sentido e interpretar las interacciones en el aula.

Nuestro objetivo es explorar como podemos ayudar al profesorado en formación a adquirir competencias docentes profesionales Proponemos tres aspectos clave para reconocer una visión profesional (Jacobs, Lamb, \& Philipp, 2010; Sherin \& Van Es, 2002): 
1. Determinar lo que es importante o significativo de una situación de clase.

2. Establecer las conexiones entre los aspectos específicos de las acciones de clase y los principios generales de la enseñanza y el aprendizaje que representan.

3. Razonar acerca de interacciones en el aula, tomando decisiones de acción.

La capacidad de los docentes de mirar e interpretar las interacciones de clase es algo que se desarrolla en el transcurso del tiempo. Afirmamos que la formación docente debe apoyar al profesorado en formación en el aprendizaje a mirar con sentido, identificando las situaciones interactivas e interpretándolas.

Entendemos aprender a enseñar "como un proceso por el cual las personas se apropian en un contexto social de instrumentos para pensar y actuar en una comunidad de práctica" (Llinares \& Valls, 2009). Así, se hace necesario que los estudiantes para profesores tengan la posibilidad de aprender, desde su práctica situada, en las intervenciones matemáticas que tienen lugar durante su proceso de prácticas escolares enseñando matemáticas.

Los tres aspectos clave de la visión profesional implican ser competente en las habilidades de identificar, interpretar y saber razonar los distintos enfoques o estrategias que el alumnado puede usar en la resolución de problemas. Además, incluye el saber orquestar todas estas producciones del alumnado. El desarrollo profesional de un profesor con estas competencias docentes puede facilitarse en un contexto instruccional formativo en el que puedan ejercitarse de manera específica y disciplinada estos aspectos docentes. Los contenidos de didáctica apoyaran este proceso de desarrollo competencial.

Desde esta perspectiva se hace imprescindible que los estudiantes puedan tener los datos suficientes en forma de videos, interacciones orales $\mathrm{y}$ escritas, diarios $\mathrm{y}$ cuestionario final, para revisar sus actuaciones y reflexionar sobre ellas.

\section{Experimento de enseñanza}

Con la finalidad de ayudar al profesorado en formación a desarrollar la habilidad de mirar con sentido, es decir identificar, interpretar y validar las interacciones en el aula, utilizamos el vídeo como un instrumento eficaz para este fin. El vídeo ofrece la oportunidad a los profesores de analizar un conjunto de prácticas realizadas en el aula. En primer lugar, muestra un registro permanente de las interacciones. Puede ser pausado y rebobinado por segmentos que se pueden volver a ver en múltiples ocasiones, con diferentes perspectivas. Esto permite a los profesores elegir segmentos específicos basados en una meta en particular. Por ejemplo, un profesor puede ver varios segmentos de un alumno y examinarlo de cerca. $\mathrm{O}$ un estudiante para profesor puede seleccionar el discurso de los alumnos y encontrar todas las instancias del discurso de alumno a alumno.

Destacamos que el hecho de disponer de una competencia docente o de una visión profesional no es una descripción de unas actuaciones, sino la capacidad de identificar los aspectos matemáticos en referencia a los contenidos, las competencias matemáticas que se pueden o no producir en la implementación de cada una de las unidades didácticas que los estudiantes han llevado a cabo y poder dotarlas de sentido. Es decir, interpretarlas desde un posicionamiento teórico determinado. Además, disponer de una visión profesional nos debe permitir identificar y sobre todo interpretar las interacciones que se producen en la clase, entre los propios alumnos y el profesor. Para identificar lo relevante se deben proporcionar instrumentos y documentación al profesorado en 
formación para ayudarles en la identificación o visión profesional de la que debe disponer un docente. Esta forma de mirar nos puede conducir a transiciones en el desarrollo profesional, incorporando reflexiones y análisis de la práctica de los profesores que enseñan matemáticas.

En la formación inicial de profesores de primaria o secundaria los estudiantes para profesor deben realizar unas prácticas (practicum), distribuidas en dos períodos. En el primer período se lleva a cabo una observación, centrada en la dinámica general de la clase, en su gestión y participando en la gestión de algunas actividades. Resaltaremos que sus observaciones se centran de manera particular en el tutor/a de clase, teniendo en cuenta su planificación y la posterior reflexión, en ellas se recogen:

- Las actividades matemáticas que se realizan,

- Cómo se realizan,

- La gestión que se produce en el planteamiento de las actividades como en el desarrollo,

- Qué tipo de intervenciones y

- Actuaciones que tienen lugar cuando se enseñan matemáticas.

De esta manera los estudiantes para profesores pueden recoger una información real sobre la práctica de enseñar matemáticas. La reflexión de los estudiantes en prácticas nos sitúa en un entorno de formación profesional, donde se preparan las actividades o tareas, para su posterior implementación en el aula, incorporándose a un proceso de introducción en una comunidad constituida por la práctica de enseñar matemáticas. Una comunidad que comparte tareas, así como la generación y uso de determinados instrumentos. Todo este proceso desemboca en la presentación de la unidad didáctica, organizando y planificando el contenido matemático para enseñarlo, el conjunto de actividades o tareas y la gestión matemática que tendrá lugar cuando intervenga y actúe con los alumnos (ver Tabla 1).

El segundo período, corresponde a la intervención y la implementación de su unidad didáctica; los estudiantes deben elaborar un diario que recoja toda la información de sus actuaciones, así como las primeras reflexiones sobre el funcionamiento de sus intervenciones. Al mismo tiempo, se efectúa la grabación de las diferentes intervenciones del estudiante para profesor con los alumnos en dos situaciones diferenciadas: una interviniendo en pequeño grupo y otra con el gran grupo. Los estudiantes recogen las actividades que sus alumnos de primaria o secundaria realizan para poder analizarlas e interpretarlas. Con este planteamiento pretendemos recoger la actuación de los alumnos de primaria o secundaria y las intervenciones que realizan los estudiantes para profesores, de manera que disponemos de datos que podrán potenciar la reflexión y el análisis de su práctica como profesores de primaria o secundaria enseñando matemáticas. Este diseño instructivo es lo que llamamos videorelato. Por tanto, los datos que tendremos en cuenta para la reflexión y el análisis son los diferentes diarios de los estudiantes, los videos que se han grabado de las dos situaciones anteriormente enunciadas, y las distintas reflexiones que cada uno presenta después de las intervenciones. 
Tabla 1. Propuesta de fases iniciales de trabajo.

\begin{tabular}{|c|c|c|c|}
\hline Fases & Contenidos & Acciones & Evaluación \\
\hline 1 & $\begin{array}{l}\text { - Análisis y } \\
\text { comparación de } \\
\text { dos sesiones de } \\
\text { clase de } \\
\text { diferentes } \\
\text { áreas. }\end{array}$ & $\begin{array}{l}\text { Discusión sobre la descripción de la situación } \\
\text { de enseñanza y su respectiva interpretación y } \\
\text { reflexión. Esta discusión puede presentarse en } \\
\text { Power Point de forma que permita comunicar } \\
\text { y sintetizar las reflexiones. } \\
\text { - Buscar los puntos de acuerdo y desacuerdo } \\
\text { entre cada una de las presentaciones. } \\
\text { - Plantear la cuestión: En función de la } \\
\text { discusión, ¿cuáles son las competencias que se } \\
\text { deben contemplar, desde la perspectiva de un } \\
\text { maestro/a enseñando matemáticas? }\end{array}$ & $\begin{array}{l}\text {-Señalar las } \\
\text { diferentes maneras de } \\
\text { actuar: } \\
\text { Semejanzas y } \\
\text { diferencias. } \\
\text { - Llenar las tablas } \\
\text { "Cómo trabaja las } \\
\text { actividades" con } \\
\text { ilustraciones. } \\
\text {-Documento de } \\
\text { síntesis de la } \\
\text { discusión. }\end{array}$ \\
\hline 2 & $\begin{array}{l}\text { - Diseño de una } \\
\text { unidad } \\
\text { didáctica: una } \\
\text { construcción } \\
\text { poliédrica. } \\
\text { - Competencias } \\
\text { básicas. }\end{array}$ & $\begin{array}{l}\text { - Discutir el documento sobre las } \\
\text { competencias del maestro/a enseñando } \\
\text { matemáticas. } \\
\text { - Elaborar un documento dónde se recojan las } \\
\text { modificaciones o no, con respecto al anterior. } \\
\text { - Elaborar un informe sobre la evaluación que } \\
\text { realiza, teniendo en cuenta los siguientes. } \\
\text { aspectos: ¿Qué evalúa?; ¿Cómo evalúa?; ¿Para } \\
\text { qué evalúa?; ¿Qué instrumentos utiliza para } \\
\text { evaluar?; ¿Cómo gestiona la evaluación? }\end{array}$ & $\begin{array}{l}\text { - Entrega del } \\
\text { documento sobre las } \\
\text { competencias del } \\
\text { maestro dónde se } \\
\text { recojan las } \\
\text { modificaciones, de } \\
\text { manera justificada, } \\
\text { argumentada. } \\
\text { - Elaborar un informe } \\
\text { sobre la evaluación. }\end{array}$ \\
\hline
\end{tabular}

Para poder realizar las reflexiones, los análisis y las posibles interpretaciones de cada una de las actuaciones que se han llevado a cabo por los estudiantes, se entregan documentos sobre competencias docentes del maestro y enseñanza de las matemáticas, donde quedan recogidos los posibles marcos de referencia para poder desarrollar las explicaciones, justificaciones $\mathrm{y}$ argumentaciones de los posibles cambios $\mathrm{o}$ modificaciones que se producen en las prácticas, en definitiva la construcción de conocimiento profesional a partir de su práctica. Como medida para reforzar este proceso los estudiantes pueden usar otros documentos, algunos entregados en el primer período, que les permita justificar y argumentar el discurso en relación con sus cambios.

Se les proporciona también un guión, para facilitar que centren su mirada para interpretar con sentido, tal como se muestra en la Figura 1:

1. ¿Presentamos la información de forma diversa (textos, gráficos, datos, cuadros...)?

2. ¿Hacemos preguntas dirigidas a comprobar la comprensión de las actividades propuestas?

3. ¿Trabajamos estrategias de comprensión de la información, como por ejemplo:

a. La identificación del que se pide a la actividad.

b. La distinción de la información relevante.

c. La interpretación de los datos.

d. Establecimiento de relaciones con conocimientos y actividades trabajados anteriormente?

4. ¿Pedimos una explicación de las actividades propuestas como paso previo a su resolución?

5. ¿Pedimos que los alumnos propongan actividades parecidas a partir de una noticia, unos datos, un dibujo u otros elementos del entorno cotidiano?

Figura 1. Cómo trabajar la comprensión de las actividades. 
Llegado a este punto, los estudiantes tienen que escoger del conjunto de las actividades que han realizado, una actividad para ser presentada y analizada con todos sus compañeros estudiantes, dando lugar a una interacción entre los estudiantes. Esta aporta al conjunto de los participantes ideas diferentes, nuevos recursos, aportaciones de nuevas actividades, de materiales que pueden ser utilizados, etc. de intercambio de significados, de maneras de actuar, en definitiva modificaciones y mejoras en relación a su intervención y en cuanto a la generación de nuevo conocimiento como profesor de primaria o secundaria enseñando matemáticas.

La siguiente etapa de reflexión y análisis de sus intervenciones se produce a partir de la selección de segmentos de la grabación que identificaremos como momentos de aprendizaje (ver Tabla 2), como puntos esenciales de una conversación que tienen una naturaleza propia y un potencial de cambio específico en el desarrollo de los participantes en la realización interactiva de las actividades escolares, didácticomatemática de un fragmento de una clase.

Tabla 2. Propuesta de fases finales del practicum

\begin{tabular}{|c|c|c|c|}
\hline Fases & Contenidos & Acciones & Evaluación \\
\hline 3 & $\begin{array}{l}\text { - Momentos de } \\
\text { aprendizaje. }\end{array}$ & $\begin{array}{l}\text { Presentación de los diferentes momentos de } \\
\text { aprendizaje. } \\
\text { Analizar cada uno de los momentos } \\
\text { presentados desde el marco de referencia de } \\
\text { los diferentes artículos, para su justificación } \\
\text { y argumentación (en relación con las } \\
\text { variables de gestión, diseño instructivo y } \\
\text { temático, uso de recursos y } \\
\text { aprovechamiento de la interacción social). }\end{array}$ & $\begin{array}{l}\text { Presentación } \\
\text { argumentada del } \\
\text { documento de las } \\
\text { valoraciones y las } \\
\text { conclusiones. }\end{array}$ \\
\hline 4 & $\begin{array}{l}\text { - Compromiso de } \\
\text { mejora profesional. }\end{array}$ & $\begin{array}{l}\text { Presentación de propuestas de cambio o de } \\
\text { modificación en relación con los diferentes } \\
\text { momentos de aprendizaje - ¿Cómo se } \\
\text { traducirían estas modificaciones en la } \\
\text { práctica? }\end{array}$ & $\begin{array}{l}\text { Presentación del } \\
\text { documento de las } \\
\text { modificaciones. }\end{array}$ \\
\hline 5 & $\begin{array}{l}\text { - Desarrollo } \\
\text { profesional. }\end{array}$ & $\begin{array}{l}\text { Debatir qué conocimientos he incorporado } \\
\text { como profesional que impartiré matemáticas } \\
\text { en primaria, a partir de la práctica realizada } \\
\text { en mis intervenciones y considerando todo } \\
\text { el proceso desarrollado. Debate. }\end{array}$ & $\begin{array}{l}\text { Contestar al } \\
\text { cuestionario final } \\
\text { Entrega de la } \\
\text { carpeta de } \\
\text { formación. }\end{array}$ \\
\hline
\end{tabular}

Se presenta a los estudiantes ejemplos de cómo deben ser seleccionados los momentos de aprendizaje que pueden identificar en una sesión:

- Ayuda y explicaciones entre compañeros.

- Explicación de la resolución de una alumna.

- Explicación de la resolución a partir de un dibujo.

- Explicación al grupo de clase de un compañero por medio de una tabla.

- Estableciendo relaciones entre diferentes estrategias. 


\begin{tabular}{|c|c|}
\hline \multicolumn{2}{|c|}{ Modelos de estudiantes: Momento de reformulación de un concepto matemático } \\
\hline & $\begin{array}{l}\text { Justificación: } \\
\text { He escogido este momento porque durante la clase no fui } \\
\text { consciente y desencadena un diálogo interesante dentro del } \\
\text { grupo. Fue cuando visualizaba el vídeo que pude observar de } \\
\text { qué manera había reconducido algunas de las observaciones del } \\
\text { grupo protagonista. }\end{array}$ \\
\hline $\begin{array}{l}\text { Descripción: } \\
\text { Durante la observación en } \\
\text { pequeños grupos de las dos } \\
\text { balanzas fui haciendo preguntas } \\
\text { para guiar aquellas aportaciones } \\
\text { que los alumnos iban diciendo. } \\
\text { En un grupo, pregunté qué habían } \\
\text { observado en la balanza mono } \\
\text { plato y me contestaron dónde se } \\
\text { leía el peso y qué era el peso } \\
\text { máximo que podía leer la } \\
\text { balanza. Mi actitud me llevó a } \\
\text { enfatizar esta aportación. }\end{array}$ & $\begin{array}{l}\text { Análisis: } \\
\text { Un alumno hace una aportación sobre el funcionamiento de la } \\
\text { balanza a partir de mi explicación sobre el equilibrio de los dos } \\
\text { platos y reconstruye la idea de igualdad entre las masas de los } \\
\text { dos platos. Mediante esta aportación, reformulo este comentario } \\
\text { para poder explicar el funcionamiento de la balanza en general } \\
\text { a toda la clase y potenciar el concepto de igualdad que hay } \\
\text { detrás, que hasta ahora no se había verbalizado. Con esta } \\
\text { intervención intento estructurar los conceptos matemáticos que } \\
\text { han aparecido espontáneamente en el diálogo en el gran grupo, } \\
\text { dirigir la reflexión a todos los alumnos. ¿Creéis que es } \\
\text { conveniente estructurar los conceptos emergentes por la vía de } \\
\text { intervenciones del maestro? }\end{array}$ \\
\hline
\end{tabular}

Figura 2. Ejemplos de momentos de aprendizaje realizados por los estudiantes

Este tipo de comentarios se encuentra en el nivel de reflexión e interpretación de la situación de práctica que permita evaluarla, tanto desde el punto de vista de la actuación de los alumnos, como la actuación que el estudiante para profesor realiza. Hay una incorporación del contenido matemático y su aprendizaje. Al mismo tiempo las interacciones que se producen entre los estudiantes favorecen el proceso comunicativo, argumentativo y reflexivo sobre sus actuaciones.

Estos modelos son elementos instructivos de un diseño formativo, que permite obtener registros de la práctica de los estudiantes para profesor, tanto a nivel escrito como audiovisual. Con la discusión y debate de estos registros, junto con los informes reflexivos, se ejercitan las habilidades de la competencia docente de identificar, interpretar y razonar o validar las interacciones en el aula. Se remarca el hecho de que las tareas de cada video-relato, implican la descripción, justificación, y análisis y reflexión por parte de cada estudiante para profesor. Estos registros, como hemos dicho son compartidos y debatidos en un entorno formativo, tanto en sesiones presenciales como virtuales. Todas estas tareas son aspectos para evaluar de forma continuada (véase la columna de evaluaciones de las Tablas 1 y 2), tienen como misión favorecer y determinar en qué medida los estudiantes en formación pueden desarrollar las habilidades de las competencias docentes que hemos sistematizado en las acciones cognitivas de identificar, interpretar y razonar sobre las estrategias de aprendizaje, observadas en las sesiones de clases del alumnado. 


\section{Diseño de la investigación}

\subsection{Datos}

Durante el año académico 2009-2010, se realizó un estudio con un grupo de estudiantes del $3^{\circ}$ de la especialidad de primaria y del máster de formación de profesores de secundaria. Los datos de esta investigación proceden de la participación de los estudiantes para profesor en entornos de formación. Estos entornos integran registros desde la práctica, documentos teóricos, espacios de interacción social cuyas actividades exigen realizar síntesis de las interpretaciones. Son tareas-casos construidas desde registros de la práctica, procedentes de video-relatos de clases, elaborados por ellos mismos a partir de sesiones del prácticum con alumnos de primaria y secundaria resolviendo problemas de matemáticas.

Se realiza un análisis de las participaciones en los debates e informes escritos, considerando en qué forma los estudiantes en formación son capaces de identificar e interpretar las estrategias usadas por los alumnos de primaria y secundaria, y cómo valoran las decisiones de las orquestaciones en clase, en especial en los momentos de puesta en común del tutor, indicando en qué medida se identifican las ideas matemáticas significativas en cada estrategia.

Los estudiantes para profesores observan a los profesores de aula que son los tutores de prácticas respectivos. En general sus observaciones se centran en las actuaciones que los profesores llevan a cabo con sus alumnos y de manera concreta en los aspectos que hacen referencia a la gestión de las actividades que desarrollan, el tipo de intervención que el profesor realiza con los alumnos, tanto en el planteamiento de la actividad o tarea matemática que se lleva a cabo, como en la gestión que se hace de ella, tal como se muestra en los escritos y comentarios expuestos por los estudiantes.

\subsection{Análisis}

La visión profesional de los estudiantes para profesor puede ser analizada en dos niveles. Un nivel se corresponde con el tipo de análisis en el que los profesores identifican acontecimientos dignos de mención, comunican principios específicos de la enseñanza y el aprendizaje, e interpretan los acontecimientos que identificaron. En otro tipo de análisis, de un nivel superior, los profesores empiezan a establecer conexiones entre lo que hacen y conceptualizan de los principios de la enseñanza/aprendizaje y proporcionan alternativas pedagógicas basadas en sus interpretaciones.

Consideraremos que se ha producido un refinamiento reflexivo, cuando haya evidencias de estrategias nuevas, adaptando y mejoras en el punto de vista. Podemos considerar que se produce un nivel alto al relacionar los aportes de la didáctica de la matemática en cuanto a la verbalización, favorecer la comunicación, potenciando la interacción entre los alumnos y el profesor.

Un rasgo característico que es muy importante para los estudiantes, es el hecho de escuchar a los alumnos, estar abierto a qué hacen y dicen para poder interpretarlo desde la matemática y su didáctica; de esta manera sus intervenciones pueden incidir en sus discursos, significados y en las actuaciones, y al mismo tiempo permite una regulación. Este aspecto incorpora la reflexión y el análisis de las producciones de los alumnos, de las explicaciones y argumentaciones, lo cual nos indica un índice de profesionalización. 
El análisis seguirá un proceso inductivo de generación de categorías que pueden esquematizarse en la Tabla 6, a partir de los registros proporcionados por los estudiantes en formación con la finalidad de valorar los rasgos característicos de la competencia docente del sentido o visión profesional en el pensamiento matemático de los alumnos en los diferentes ámbitos del contenido matemático considerado.

\section{Un marco para el análisis de las prácticas}

Asumimos que el tipo de diseño formativo o prácticum, que hemos presentado, representa un profundo análisis de las prácticas escolares organizadas en torno a principios generales de enseñanza y aprendizaje. Se incluyen conexiones entre los detalles del análisis del segmento de clases y los conceptos y principios en que están involucrados. Hablar de su enseñanza en términos de estos trozos de análisis sugiere que los profesores pueden identificar lo que es importante en un segmento de la enseñanza y no simplemente recordar lo que ocurrió paso a paso. De esta manera, los estudiantes para profesor pueden desarrollar una visión más profesional para discriminar las interacciones significativas de la clase.

Para evaluar el grado de visón profesional que se pueda alcanzar, hemos creado una trayectoria de los niveles de desarrollo del aprendizaje para identificar e interpretar las interacciones en el aula (Tablas 3, 4, 5).

Lo que sigue es una descripción de cada uno de los niveles con ejemplos extraídos de prácticas escolares (Tabla 6) considerando tres categorías:

1. Tipo de competencia matemática.

2. Reconocimiento una visión profesional.

3. Indicadores de calidad de desarrollo.

Tabla 3. Tipos de competencia matemática

\section{Tipo de competencia matemática}

Competencia matemática.

Comprensión conceptual.

Procesos: construcción utilizando y explicitando conocimientos de propiedades y elementos.

Procedimientos, comunicar .

Identificar estructuras generales, ...

Actitudes.

Gestión del profesor:

Pedir generalizaciones del procedimiento seguido.

Establecer normas relativas al aprendizaje.

Seleccionar "tareas" matemáticas importantes que

faciliten el desarrollo de las diferentes

dimensiones de la competencia matemática.

Guiar la discusión y la comunicación.
Aspectos de la Enseñanza .

Tarea: demanda cognitiva/ favorecer la comunicación.

Interacción: alumnos presentan y explican, analizan y comparan estrategias.

Inclusión de argumentos matemáticos en las explicaciones y no sólo descripción de procedimientos.

Normas sociales - potenciar el que los estudiantes se sientan libres de expresar sus ideas. 
Tabla 4. Visión profesional

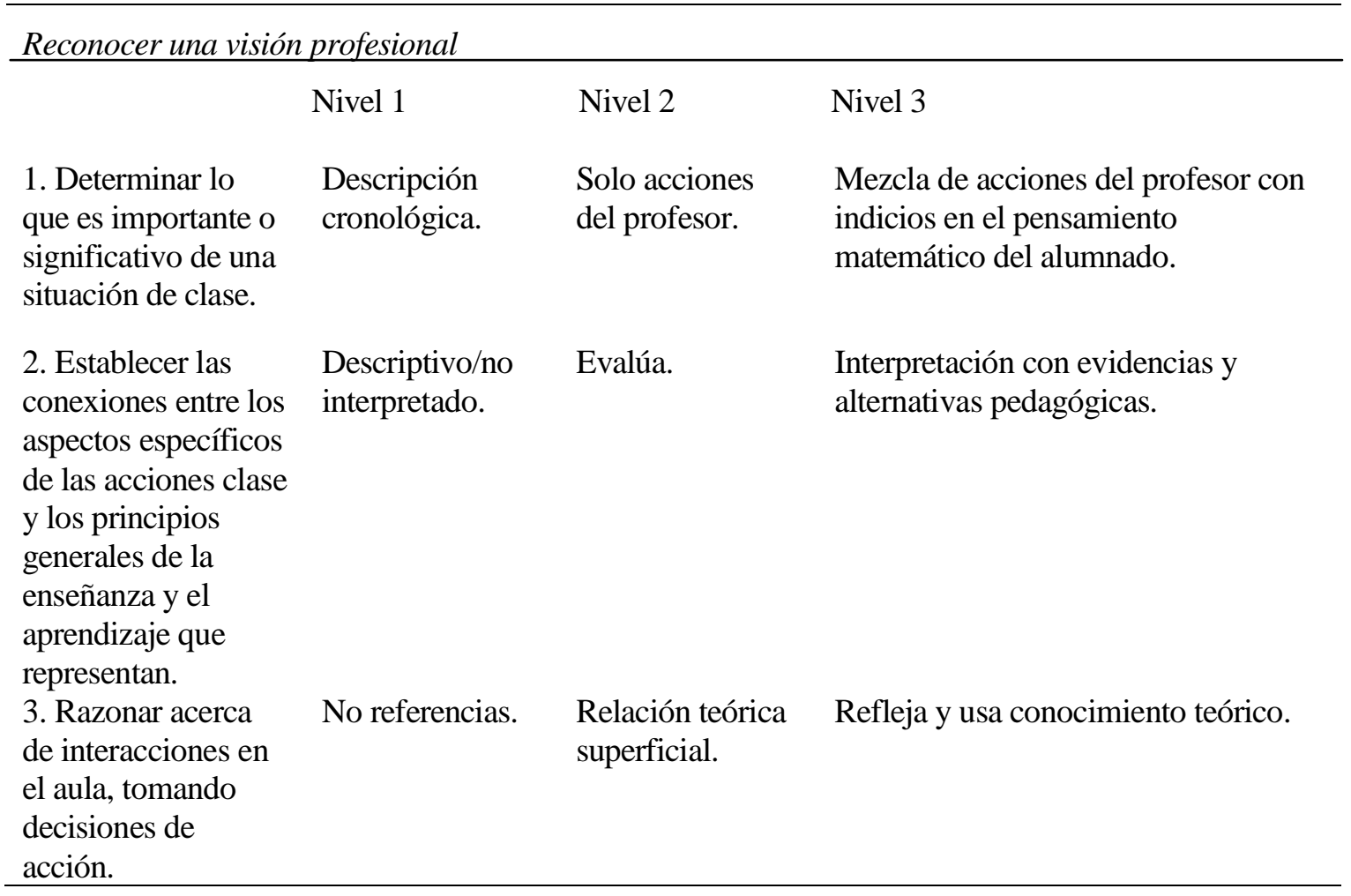

Tabla 5. Dimensiones categóricas para interpretar las interacciones en el aula

\begin{tabular}{|c|c|c|}
\hline \multicolumn{3}{|c|}{ Escalas de Graduación de los indicadores de calidad de desarrollo } \\
\hline Niveles. & $\begin{array}{l}\text { Uso de nuevas } \\
\text { estrategias } \\
\text { (cambio didáctico). }\end{array}$ & $\begin{array}{l}\text { Uso de nuevas epistemologías } \\
\text { (cambio conceptual). }\end{array}$ \\
\hline 0 & No usa. & $\begin{array}{l}\text { No tiene nueva perspectiva ni se } \\
\text { implica. }\end{array}$ \\
\hline Reconocimiento: I/II. & $\begin{array}{l}\text { Tiene un conocimiento } \\
\text { naif/competente. }\end{array}$ & $\begin{array}{l}\text { Reconoce conocimientos sin } \\
\text { integrarlos. }\end{array}$ \\
\hline Ejecución heurística: III. & $\begin{array}{l}\text { Reconoce estilos de } \\
\text { aprendizaje. Los usa, } \\
\text { pero mecánicamente. }\end{array}$ & $\begin{array}{l}\text { Tiene una comprensión } \\
\text { naif/satisfactoria. Intenta integrar los } \\
\text { nuevos conocimientos a su estilo. }\end{array}$ \\
\hline Practicidad (rutina analítica): IV. & $\begin{array}{l}\text { Ha incorporado como } \\
\text { propia rutina las nuevas } \\
\text { estrategias. Planifica y } \\
\text { emite juicios coherentes. }\end{array}$ & $\begin{array}{l}\text { Capacidad analítica mínima } \\
\text { Integración conceptual aceptable. } \\
\text { Ha modificado su estilo de } \\
\text { enseñanza acorde con las nuevas } \\
\text { epistemologías. }\end{array}$ \\
\hline
\end{tabular}

Hablar de descriptores de niveles, implica asumir que hay un desarrollo, es decir, aprendizaje de algo. En el ámbito del desarrollo de una mirada con sentido, como un aspecto de la competencia docente, este desarrollo no es ingenuo, sino que se manifiesta por la integración de las ideas provenientes de la didáctica de la matemática en el discurso generado cuando realizan las justificaciones de las identificaciones de los 
hechos relevantes y proponen interpretaciones. Para poder dar sentido a los descriptores de los niveles de desarrollo de la competencia profesional e identificar la calidad en las interacciones en el aula, suponemos que los profesores en formación tienen información proveniente de la didáctica de las matemáticas, sobre estilos de aprendizaje e epistemologías correspondiente a una diferenciación entre los diferentes paradigmas: transmisivo, de comportamiento y socio-constructivista, de los contenidos de aprendizaje sobre la práctica docente y el currículo de las matemáticas escolares.

\section{Resultados y discusión}

En la evaluación de esta propuesta formativa se recogen diferentes niveles de identificación, interpretación y análisis que los estudiantes para profesor realizan. Se identifican las evidencias que se producen entre los propios alumnos y el tutor de clase, se contemplan los contenidos matemáticos y la gestión que se está realizando. Estos se han elaborado a partir de las tres dimensiones consideradas: Tipo; Niveles y Desarrollo profesional.

Se ha pretendido generar a partir de este análisis, categorías de relaciones entre las formas de participar y los niveles de desarrollo de los procesos de interpretación del aprendizaje de las matemáticas vinculados a ámbitos específicos del conocimiento matemático escolar.

En la Tabla 6 se recogen de manera sintética algunas evidencias.

Hay poca observación que atienda a lo que los alumnos dicen, aunque se anuncian las situaciones en que se producen interacciones entre los alumnos pero sin describir qué se dice en ellas:

"Hacen un ejercicio de descomponer la decena, el ejercicio constaba de una suma de tres números y los alumnos habían de explicar como hacían la suma. En este caso creo que el tutor de prácticas quería observar cuáles eran las estrategias usadas por los alumnos"

La anotación de este estudiante intenta interpretar lo que el tutor de prácticas pretende con este tipo de actividad, pero sin centrarse en lo que los alumnos pueden estar diciendo cuando realizan la actividad, de manera que no se referencia el pensamiento matemático que los alumnos pueden mostrar en la interacción.

Por parte de algún estudiante se nos comunica en su descripción que se produce una interacción entre los alumnos en el desarrollo de una tarea:

"Los alumnos habían de construir una especie de modelo de una tabla de multiplicar . . . Para construirla pidió a los alumnos que pensasen como podían hacerlo. Y después los alumnos pusieron en común las diversas soluciones a la pregunta. Entre todos llegaron a la conclusión que la mejor solución era dividir, midiendo con la regla la cartulina".

En ella no se recoge lo que piensan matemáticamente, de manera que se apunta la interacción pero no se informa sobre lo que en ella se dice.

También se recoge cómo funcionan las clases de matemáticas, lo que hace referencia a la gestión y al mismo tiempo a cuestiones de carácter pedagógico:

"La mayoría de trabajos que se hacen en la clase de matemáticas son de tipos individual (problemas de operaciones simples, trabajos de lógica...) pero el tutor de prácticas, cuando todos han acabado el trabajo, hace una puesta en común sobre que se había de hacer, como lo han hecho y por qué lo han hecho de esta manera y no de otra. Esta metodología favorece la comunicación entre los alumnos i estos pueden ver que no hay un único proceso para llegar a la solución".

Se evalúa este proceso sin dar una justificación, de manera que se tiene en consideración, sin una interpretación que nos indique el contenido que pueden expresar los alumnos en la puesta en común. 
Tabla 6: Extractos de prácticas escolares

Niveles de análisis de Nivel profesional
la enseñanza

\begin{tabular}{|c|c|}
\hline Tipo & Datos \\
\hline \multicolumn{2}{|c|}{ Aspectos de la Enseñanza } \\
\hline $\begin{array}{l}\text { Propuesta de } \\
\text { trabajar con los } \\
\text { alumnos con } \\
\text { recursos } \\
\text { gráficos. }\end{array}$ & $\begin{array}{l}\text { "quería hacerte un comentario sobre las estrategias. } \\
\text { No sé si recuerdas la LNV la línea numérica vacía. La } \\
\text { LNV es un modelo estructurador que permite a los } \\
\text { alumnos representar de forma gráfica las diferentes } \\
\text { estrategias utilizadas...” }\end{array}$ \\
\hline $\begin{array}{l}\text { Favorecer los } \\
\text { procesos de } \\
\text { generalización } \\
\text { explícita. }\end{array}$ & $\begin{array}{l}\text { "En tercer lugar, se podrían haber utilizado actividades } \\
\text { generales y partiendo de la reflexión de mis valores } \\
\text { posibles, imposibles se puede deducir la relación } \\
\text { general que se estaba buscando." }\end{array}$ \\
\hline $\begin{array}{l}\text { Variable de } \\
\text { gestión del } \\
\text { aula. }\end{array}$ & $\begin{array}{l}\text {.... sé como estar dentro del aula y hacer que los niños } \\
\text { me sigan, } \\
\text {... yo me comprometo a partir de ahora, y en lo } \\
\text { sucesivo, a buscar cómo hacer que la atención se } \\
\text { mantenga durante todo el rato, y empezaré haciéndolo } \\
\text { partiendo de los alumnos, es decir, pidiéndoles a ellos } \\
\text { el porqué de su no seguimiento, para así incidir en } \\
\text { aquello que realmente los atrae y aprovecharme de esta } \\
\text { situación, lo cual me traerá a menudo a reformular las } \\
\text { actividades que tenía diseñadas previamente... }\end{array}$ \\
\hline
\end{tabular}

Solo acciones del profesor.

Interpretación con evidencias y alternativas pedagógicas.

Interpretación con evidencias y alternativas pedagógicas.

Mezcla de acciones del profesor con indicios en el pensamiento matemático del alumnado.

Gestión del profesor

Variable del ...supe aprovechar que un alumno hacía un dibujo en el uso de recursos. suelo con el dedo para explorar lo que ellos sabían del tema, puesto que al final hice que todos dibujaran con el dedo diversas figuras, lo cual les hizo tomar conciencia de las características de estas ... en un futuro en el centro dónde me envíen no cuente con demasiados recursos, esto no será una excusa para no hacer algo, puesto que yo con aquella actividad, y con muchas otras que he podido observar a Carmen, los recursos surgieron, no estaban pensados para realizar aquello, puesto que la utilidad que nosotros damos a los objetos de qué disponemos puede variar mucho según nuestro propósito.

Orquestación Una vez acabada la primera construcción de triángulos del dialogo es necesario que los alumnos expliciten el entre alumnos. procedimiento. Podrías haber regulado un diálogo entre los alumnos para ver cuáles eran las diferentes opiniones y pidiéndoles que las justificasen.
Solo acciones del profesor

Ejecución heurística. Tiene una comprensión naif/ satisfactoria. Intenta integrar las nuevas epistemologías en su estilo.

Reconoce estilos de aprendizaje. Los usa, pero mecánicamente.

Practicidad (rutina analítica) capacidad analítica mínima Integración conceptual aceptable.

Ha modificado su estilo de enseñanza acorde con las nuevas epistemologías.

Practicidad (rutina analítica capacidad analítica mínima Integración conceptual aceptable.

Ha modificado su estilo de enseñanza acorde con las nuevas epistemologías.

Ejecución heurística: Reconoce estilos de aprendizaje. Los usa, pero mecánicamente. 
La interpretación y el análisis, que nos permite obtener indicios de cambio tiene su sentido o significado en la implementación de un proceso de prácticas para estudiantes para profesores que enseñan matemáticas, tal como recoge el texto que los estudiantes escribieron:

"Porque nos han permitido observar qué tipo de maestro de matemáticas queremos ser. Primero analizar el maestro que teníamos como tutor en el centro. Este análisis de todos los maestros del grupo de prácticum nos sirvió para ver cuáles eran para nosotros los aspectos a mejorar. Después nos analizamos a nosotros mismos y a nuestros compañeros. Durante el curso hemos ido cambiando nuestra perspectiva de cómo se debe producir el proceso de Enseñanza-Aprendizaje. Hemos ido añadiendo contenidos aprendidos, maneras de hacer, contenidos didácticos para aplicar nuestro conocimiento en el aprendizaje. Hemos aprendido a tener en cuenta al alumnado y sus características y hemos intentado aplicar las mejores soluciones posibles con la ayuda de nuestros compañeros. Lo que hace falta a partir de ahora es no olvidar que debemos seguir trabajando por añadir cambios que nos hagan más profesionales como maestros de matemáticas".

Nos parece interesante considerar la utilización de los medios tecnológicos en el proceso de la implementación del proceso de prácticas, para su posterior utilización, para ser interpretado y analizados por los estudiantes, tal como se recoge en el siguiente comentario:

"Uno de los aspectos que me gustaría destacar es la utilización de recursos audiovisuales y tecnológicos para la mejora educativa, tanto en la universidad como en las escuelas. Creo que es importante que los docentes sean capaces de utilizar correctamente cuántas más herramientas mejor porque puedan mejorar los procesos de aprendizaje y conseguir una mejor profesionalización de nuestra tarea. En nuestro prácticum hemos utilizado el vídeo por grabar nuestras prácticas, pero también nos hemos servido de diferentes aplicaciones 2.0 como plataforma por guiar nuestro aprendizaje cooperativo. Creo que es importante potenciar desde las universidad todas estas herramientas tecnológicas que nos ayudan a ser más eficaces en nuestra tarea. Finalmente agradecer a todo el mundo por sus intervenciones, he aprendido mucho de todo el mundo y ha sido muy enriquecedor poder contrastar mi punto de vista".

En definitiva se han dado las condiciones para que los estudiantes para profesores enseñando matemáticas dispusieran de los instrumentos de video relatos que permiten "pensar y actuar", focalizando su interpretación y análisis del proceso de aprender que sus alumnos realizan, negociando los diferentes significados que en la interacción se producen, originando indicios de cambio en la calidad de desarrollo profesional, como aporta la reflexión de una estudiante:

"Creo que las prácticas que he realizado sí que han provocado un cambio en mi propia concepción del proceso de enseñanza - aprendizaje puesto que a lo largo de todo el proceso de realización y observación de las prácticas ha implicado necesariamente hacer un análisis efectivo de la situación del conocimiento del alumnado de Primaria puesto que ha de llegar a conocer y utilizar los diferentes elementos de las formas geométricas según mis propósitos iniciales reflejados en mi Unidad Didáctica. Para lo cual he utilizado los conocimientos de didáctica de las matemáticas, sobre el aprendizaje matemático para diagnosticar y dotar de significado las producciones del alumnado, identificando posibles causas que las justifiquen y proponer justificaciones y procesos de intervención. En momentos donde hay conflictos cognitivos matemáticos en el aula debo tomar decisiones sobre el diseño de situaciones de enseñanza- aprendizaje donde intervengan varios contextos para ampliar y analizar estas situaciones y así inferir un conocimiento matemático más competente. Para lo cual hace falta utilizar los conocimientos sobre el contenido matemático como objetivo de enseñanza- aprendizaje, sobre los problemas como instrumento de aprendizaje o sobre el aprendizaje para diseñar, seleccionar, analizar y modificar secuencias de enseñanza previamente establecidas. Por lo tanto, sí que hemos cambiado algo el proceso de enseñanza- aprendizaje para qué vaya dirigido hacia el análisis y la interpretación de las producciones matemáticas del alumnado".

Esta situación inicial del prácticum, que hace referencia a un profesor dando clase de matemáticas con alumnos, nos pone de manifiesto que los estudiantes para profesores 
describen en sus observaciones aspectos sobre la gestión de cómo se presentan las actividades, cuestiones de pedagogía general, si se trabaja en grupo o individual, la dinámica del funcionamiento de la clase haciendo matemáticas, y en escasas ocasiones centrándose en el pensamiento matemático de los alumnos cuando se producen las interacciones entre ellos y el profesor, de manera que no se muestran evaluaciones, ni interpretaciones del pensamiento matemático de los alumnos, aunque se nos explicite que hay interacciones.

Este proceso se modifica con la incorporación de los videos, los escritos de las interacciones que se han producido después de haber visionado los diferentes momentos de aprendizaje expuestos por los estudiantes, y las discusiones de los estudiantes y el tutor de prácticas, los diarios de las intervenciones realizadas con los alumnos para finalizar con las reflexiones - conclusiones de su proceso de práctica de enseñar.

En esta interpretación y reflexión de la práctica de enseñar matemáticas contamos con las grabaciones de video, los escritos de las interacciones que se han producido después de haber visionado los diferentes momentos de aprendizaje (vídeo-relatos) expuestos por los estudiantes para profesor en los seminarios de debate del practicum, los diarios de las intervenciones realizadas con los alumnos de primaria y secundaria, y con las reflexiones conclusiones de su proceso de práctica de enseñar.

El hecho de que los estudiantes para profesor puedan utilizar el video, como un estudio de casos, y en particular, de su caso, confirma la hipótesis planteada en Llinares y Valls, (2009): de que "es la reflexión sobre y el análisis de la práctica de enseñar matemáticas la que crea las condiciones para la construcción del conocimiento útil para enseñar matemáticas" (p. 9).

Este entorno formativo, aquí considerado, provoca cambios en lo didáctico y en lo profesional. Se hace evidente que los estudiantes para profesor hablan, y escriben, teniendo en cuenta que pueden hacer referencia al esfuerzo, a la construcción de conocimiento, al trabajo en grupo, y a los cambios que nos permitirá una mejor interpretación sobre los indicios de cambio. Se produce un nivel de refinamiento reflexivo, incorporando, adaptando y mejorando su punto de vista profesional.

Tal como mostramos en la Tabla 6 se produce un cambio en las concepciones que los estudiantes tienen en referencia a sus intervenciones, en la práctica de enseñar matemáticas, siendo capaces de reflexionar a partir de su práctica situada. Incorporan en sus reflexiones todos sus conocimientos y por medio de la reflexión y el visionado de sus actuaciones son capaces de iniciar un proceso de razonamiento profesional, dando lugar a la generación de su conocimiento práctico personal. Se confirma que el proceso de interacción que establecen los estudiantes en la presentación de los diferentes momentos de aprendizaje recogidos en la Tabla 2, influye, y que los cambios que se llevan a cabo se generan en la reflexión de su práctica. Con la participación de sus compañeros y el tutor de prácticas, se constituye una comunidad de práctica, dando lugar a un aprendizaje y configurando su significado.

En la Tabla 6 se muestra también la potencialidad de los videos relatos, para poder analizar su práctica situada, y de esta manera ser consciente de aquellos cambios que se han de producir en sus intervenciones con los alumnos, y en la gestión matemática que deben realizar para obtener de ellos un significado matemático. Esta potencialidad de los videos relatos pone de manifiesto el grado de emergencia de los conocimientos de los estudiantes y su desarrollo profesional, cuando son capaces de analizar su práctica y proponer cambios a sus actuaciones.

Tal como se recoge en el proceso de prácticas, los estudiantes en la situación inicial realizan unas observaciones que nos describen las situaciones que se llevan a cabo entre los alumnos, donde se contempla la gestión que se hace en ellas, las cuestiones de carácter pedagógico general en cuanto a las instrucciones, la dinámica que el tutor de prácticas y los alumnos establecen, cuando se producen las interacciones entre los alumnos, cómo se 
desarrolla el clima en la clase, el tipo de actividades que realizan, pero en ningún momento hay constancia de lo que dicen, cómo hablan los alumnos de matemáticas, lo que nos indica que no se produce una focalización en el pensamiento matemático de los alumnos. Esta situación se modifica al final del proceso, pues los estudiantes son capaces de describir, evaluar y al mismo tiempo interpretar las diferentes interacciones que se producen entre los alumnos, recogiendo el pensamiento matemático e interpretándolo.

Es fundamental seguir investigando sobre cómo el tipo de conocimiento y cognición del profesorado puede relacionarse directamente con la calidad de aprendizajes de sus alumnos (Tchoshanov, 2011). Desde la perspectiva de la formación del profesorado, tenemos que seguir investigando más detalladamente el cómo facilitar la interpretación de las interacciones en el aula y en particular:

i. ¿Cómo focalizar más la observación en el pensamiento del alumnado que en describir el comportamiento del profesorado?

ii. ¿Cómo pueden evaluar evidencias de lo que es irrelevante para conseguir un objetivo específico de aprendizaje?

iii. ¿Cómo facilitar habilidades para distinguir entre evidencias en la fluidez de contenidos procedimentales y de conocimientos conceptuales?

Este proceso nos reafirma que si queremos que nuestros estudiantes aprendan a mirar con sentido y sean capaces de interpretar las interacciones matemáticas del aula, es necesario, que en la formación inicial del profesorado, se incorporen situaciones donde se puedan usar videos de otros profesionales y de ellos mismos en su periodo de prácticas, enseñando matemáticas, para luego poder mejorar su desarrollo profesional.

\section{Referencias}

Jacobs, V. R., Lamb, L. L., \& Philipp, R. A. (2010). Professional noticing of children's mathematical thinking. Journal for Research in Mathematics Education, 41(2), 169-202.

Llinares, S., \& Valls J. (2009). The building of pre-service primary teachers' knowledge of mathematics teaching: interaction and online video case studies. Instrutional Science, 37, 247271.

Sherin, M. G., \& Van Es, E. A. (2002). Learning to notice: Scaffolding new teachers' interpretations of classroom interactions. Journall of Technology and Teacher Education 10(4), 571-596.

Tchoshanov, M. A. (2011). Relationship between teacher knowledge of concepts and connections, teaching practice, and student achievement in middle grades mathematics. Educational Studies in Mathematics, 76, 141-164.

\section{Referencia a los autores}

Josep M. Fortuny Facultat de Ciències de l'Educació. Universitat Autònoma de Barcelona (España).

jfortuny@uoc.edu

Rafael Rodríguez Facultat de Ciències de l'Educació. Universitat Autònoma de Barcelona (España).

Rafael.Rodriguez@uab.es 